\title{
Biopsy: Vane to Diagnosis
}

\section{Veena Shankar ${ }^{1}$ and Karthik D Yadav ${ }^{2 *}$}

${ }^{1}$ Assistant Professor, Department of Oral and Maxillofacial Pathology, Government Dental College and Research Institute, Ballari, Karnataka, India

${ }^{2}$ Assistant Professor, Department of Oral Medicine and Radiology, K.G.F College of

Dental Sciences, Karnataka, India

*Corresponding Author: Karthik D Yadav, Assistant Professor, Department of

Oral Medicine and Radiology, K.G.F College of Dental Sciences, Karnataka, India.
Received: August 13, 2020

Published: August 24, 2020

(C) All rights are reserved by Veena Shankar and Karthik D Yadav.

\begin{abstract}
A healthy oral cavity is a replication of good health. Untoward changes noticed in the oral cavity should be evaluated and confirmed to render a definitive diagnosis. Biopsy remains the gold standard to render a conclusive diagnosis in adjunct with clinical diagnosis. Biopsy helps us to assess the histological characteristic pattern of different suspected lesions with its differentiation and extent or spread depending on the type of biopsy performed. Further, a definitive diagnosis will help in early treatment planning and a better prognosis, all of which is the need of the hour. The different types of biopsies are indicated for different disease process depending on its site, location, extent, accessibility, nature and the armamentarium available. Each method has its own advantages and disadvantages.
\end{abstract}

Keywords: Biopsy; Histopathology; Armamentarium; Surgery; Lesion; Disease; Diagnosis

\section{Introduction}

A healthy oral cavity is a replication of the well-being of the individual. Any untoward changes noticed in the oral cavity should be evaluated and confirmed to render a definitive diagnosis. Not all changes are permanent and have the potential to heal or repair itself without any long term deleterious effects. However, any abnormal changes for a significant amount of time should not be left unnoticed. The observation of the tissue specimen under a microscope, taken from a living organism is termed as biopsy [1].

The term Biopsy is derived from the Greek words 'bios' and 'opsis' which means life and vision correspondingly. Biopsy is a surgical process involving procurement of a living tissue specimen, to reach a definitive diagnosis. Till date, biopsy remains the gold standard for the definitive diagnosis, by histopathologic assessment of the tissue specimen of the suspicious lesion [2].
Biopsy helps us to assess the histological characteristic pattern of different suspected lesions with its differentiation and extent or spread depending on the type of biopsy performed. Further, a definitive diagnosis will help in early treatment planning and a better prognosis, all of which is the need of the hour [1].

Biopsy also plays a very important role in establishing prognosis not only for simple lesions but also for the premalignant as well as the malignant lesions/conditions of the oral cavity. Further, prognosis helps us in determining efficacy of the treatment protocol with the morbidity as well as the mortality of the patient [3].

\section{Site selection}

Any abnormal refractory lesions persisting for more than 2 weeks, even after the removal of the etiologic agent which might be 
a simple irritation or traumatic agent to other etiologies including infective microorganisms, are strongly indicated for a biopsy $[3,4]$.

Different types of biopsy are required, according to their [4]:

- $\quad$ Site

- Depth

- Extent

- Type of tissue specimen.

The most clinically suspicious area must be the area of interest especially if any redness, an area of surface thickening or a symptomatic area is present then that is definitely the area of interest $[5,6]$.

When the lesion is multifocal or widespread leukoplakia, then multiple biopsies ('field mapping') should be considered. Lesions with the size of $<2-3 \mathrm{~cm}$, an excisional biopsy should be considered, with a smaller sized lesion, incisional biopsy should be the choice but in the case of a nonhomogeneous leukoplakia an incisional biopsy may not be representative and excision should be the best choice of diagnostic and also as an early treatment modality [6].

Further, even before biopsy, some other staining methods may be used as a diagnostic aid.

Toluidine blue is another aid which stains and helps in delineating the margins and giving the diagnostician a proper idea of the site of selection of the site of biopsy and its extent. It is a metachromatic dye belonging to the thiazine group with an affinity towards the greater than normal DNA content, especially the acidophilic components such as the carboxylates, sulfates and phosphate radicals mostly present in dysplastic and malignant cells [7].

Some studies have shown high sensitivity, no false negative and good positive predictive values [8]. However, it has also been demonstrated that toluidine blue binds to nucleic acids even in conditions such as mucosal ulcerations, formation of granulation tissue and in inflammatory lesions which gives false positive results [9].

Histopathologic examination by biopsy even to this date still remains the gold standard for diagnostic purposes [5,6]. A distinction can be made histo-pathologically between dysplastic and nondysplastic lesions in a biopsy specimen [6].
The architectural disturbance reveals the severity of dysplasia accompanied by cytological atypia. Dysplasia depicts the histological as well as changes such as the loss of uniformity or of the architecture of the epithelial cells, due to abnormal cell proliferation. However, more importance should be given that dysplasia is a spectrum and that no criteria exist which can precisely divide this spectrum into mild, moderate and severe categories [6].

Armamentarium

The minimal requirements are as follows:

- Syringe and local anesthetic

- Blade handle and no. 15 blade

- Retractor

- $\quad$ Tissue forceps

- Needle driver

- Curved scissors

- $\quad$ Sutures, if needed

- Hemostatic agents (silver nitrate or absorbable gelatin sponge)

- Gauze sponges

- Specimen bottle containing 10\% neutral buffered formalin.

\section{Indications}

1. Enlarging mass, which may be either

a. Cystic lesion: To assess the prognosis and aggressive nature which is non-characteristic of the majority of the cysts $[1,4]$.

b. Hard tissue lesions: Bone lesions accompanied by pain, sensitivity alteration or other symptoms [4].

c. Malignancy: For confirmation.

2. Oral mucosal lesions: Biopsy is strongly indicated in any lesion which show change in color, or show any kind of proliferative, ulcerative or abnormal growth [11-14].

a. Chronic ulceration,

b. Friable tissue,

c. Pigmented lesions, especially those with an irregular border and non-homogenous coloration, should be biopsied to exclude mucosal melanoma,

d. Persistent lesions: Lesions that persist for a longer time even after the removal of etiological agent $[13,14]$. 
3. Premalignant state: Suspected for malignancy [15].

4. Level of malignancy: Defining the extent of a disease process [16].

5. Idiopathic etiology: Unclear or unknown etiology [17].

6. Systemic illness: Diseases like lupus, amyloidosis, scleroderma, or Sjogren's syndrome that need a histological confirmative diagnosis [17].

7. Infectious origin; like syphilis [18].

\section{Contraindications}

1. Vascular lesions: Probability of excessive bleeding in cases of vascular lesions or damage to any of the vessels, which are richly supplied by vasculature [18]. However, a few modifications which precludes the safe use of local anesthetics [13].

2. Seriously ill patients: Risk of secondary infection [17].

3. Deep lesion: In very deep lesions in which there are chances of damage to adjacent structures [17].

4. Multiple neurofibromas: There is a risk of malignant transformation in these cases [17].

5. Esthetic reasons: Biopsy is contraindicated in lesion in which biopsy can cause esthetic changes, like the vermilion border of the lip $[19,20]$.

6. Bisphosphonate therapy: Due to risk of development of osteoradionecrosis [21].

\section{Types of biopsy}

- Incisional biopsy

- Excisional biopsy

- Punch biopsy

- Brush biopsy

- Electro-surgery and laser biopsy

- Fine needle biopsy

- Exfoliative cytology.

\section{Incisional biopsy}

It provides a representative sample of tissue for diagnostic purposes.

In case of extensive lesions, a representative sample of the lesion is removed along with the normal adjacent tissue. Further, if the lesion is extensive, then different samples should be obtained and stored separately in a proper labelled container [1].

It is relatively accurate and helps to eliminate out the other differential diagnosis [22].

\section{Advantages}

- Small tissue specimen [23]

- Less structural damage

- Size is determined by the specialist and

- Easy to involve a part of normal epithelium

- $\quad$ Easy to perform

- Better healing in comparison to excisional biopsy.

Disadvantages

- Margins may not be precise

- Non-standardized size

- Cannot be performed in small lesions ( $\operatorname{size}<0.5 \mathrm{~cm}$ )

- Depth may sometimes be insufficient

- May increase the risk of metastasis of malignant lesions

- Avoided in vascular cases as it may cause profuse bleeding [21].

\section{Excisional biopsy}

It is the complete removal of the lesion, for diagnostic as well as therapeutic purposes. Most commonly performed for small, nonmalignant, non-vascular lesions [3].

Excisional biopsy is most commonly recommended in those cases in which the size of biopsy is small [3]. Further, Small, pedunculated, exophytic lesions in accessible areas are excellent candidates for excisional biopsy $[13,14]$.

\section{Advantages}

- Complete removal of the lesion [20]

- Helps to render a diagnosis

- Provides adequate tissue for diagnosis

- Better accessibility

- Patient satisfaction.

\section{Disadvantages}

- Difficult to perform in large lesions. 
- Slow wound healing

- Aesthetics may be compromised

- Non-standardized size

- Should be avoided in cases where a high grade malignancy is suspected [21].

\section{Punch biopsy}

It may be used for either incisional biopsy or excision of a small lesion at an accessible site. The lateral tongue and buccal mucosa are appropriate sites for punch biopsy, as it must be feasible for the device to approach the mucosal surface perpendicularly.

It should not be performed in vesiculo-bullous lesions, as the rotation of the punch will detach the epithelium and prevent proper assessment of basement membrane as well as the interface between epithelium and connective tissue, which is necessary for sub-classification of such lesions.

Punch biopsy is usually used as an alternative to incision biopsies for small lesion at an accessible site. The lateral tongue and buccal mucosa are appropriate sites for punch biopsy, as it must be feasible for device to approach the mucosal surface perpendicularly [20].

\section{Advantages}

- Rapid and simple

- Inexpensive technique

- Good margins

- Good esthetics results

- Faster wound healing [1]

- Multiple samples at the same time [24]

- Less artifacts then the scalpel biopsy [2].

\section{Disadvantages}

- Highly vascularized or innervated areas cannot be biopsied

- Adequate depth may not be attained

- $\quad$ Size is limited

- Normal adjacent tissue may not be attained $[1,8]$.

\section{Electro-surgery and laser techniques [25]}

They help in maintaining a clear field with controlled bleeding. Further they are safe, easy and convenient to the patient as well as the clinician. Electro-surgery and laser techniques also produce thermal artifacts that may hamper histologic interpretation.

Lasers may be of great value, however, in managing a wound left by scalpel biopsy in areas of the mouth where closure is difficult or inappropriate. A laser produces a zone of thermal coagulation smaller than that of electro-surgery, but still, a 0.5 - $\mathrm{mm}$ margin should be maintained between the cut and the representative area to be sampled. The technique may produce good local hemostasis and minimal postoperative discomfort.

\section{Advantages}

- Less bleeding

- Highly vascularized or innervated areas can be biopsied

- $\quad$ Easy accessibility

- Better wound healing [1]

- Good margins

- Good esthetics results

- Multiple samples at the same time [24]

- Less artifacts then the scalpel biopsy [2].

Disadvantages

- Adequate depth may not be attained

- $\quad$ Size is limited

- Normal adjacent tissue may not be attained

- Thermal defects

- Bigger tissue specimen may be required.

Brush biopsy

A non-invasive method, for the evaluation of oral mucosal lesions to assess cellular dysplasia and atypia [14]. A brush biopsy was initially introduced for cervical smears in gynecological lesions and was later modified for oral smears too.

Brush biopsy is austerely done for mass screening of suspected premalignancy and malignancy and also advocated as a screening modality for innocuous lesions that may otherwise not be sampled [14].

\section{Exfoliative cytology}

OralCDx brush biopsy is an oral transepithelial biopsy system that uses computer-assisted brushing. It is the study and interpre- 
tation of the characteristics of cells that wither off, naturally or artificially from the oral mucosa.

They are of significance in monitoring several sites for a large lesion and can also guide the choice of sites for incisional biopsies. It was designed to screen innocuous looking oral epithelial abnormalities for dysplasia or cancer.

The detection of abnormal cells by this technique has a sensitivity of $52-100 \%$ and specificity $29-100 \%$. Overall, the OralCDx technique appears to overestimate dysplastic lesions and produce a high number of false-positive results $[9,27]$.

\section{Advantages}

- In contrast to exfoliative cytology, the brush biopsy collects cells from the full thickness of the oral epithelium

- Non-invasive, chair side procedure, easy to perform and painless

- Dysplasia can be ruled out

- High sensitivity and specificity

- Suspected cases of candidiasis can be rapidly confirmed through oral cavity [4].

\section{Disadvantages}

- Cannot be used as a substitute for scalpel biopsy

- Significant false finding may be observed due to sampling error [26].

Fine needle biopsy [17]

Fine Needle biopsy (FNB) is a minimally invasive technique which is particularly suitable for those sensitive areas where an incisional biopsy is contraindicated or is not possible. Although it does not provide a definite type specific diagnosis, it is used in conjunction to the clinical and radiological findings to rapidly provide the best possible initial assessment on which management decisions can be based.

\section{Advantages}

- Safe

- Inexpensive

- Rapid technique

- Accurate diagnosis
- Low risk of infections

- High index for suspicion for malignancy.

Disadvantages

- Possibility of false negative results

- $\quad$ Site precision is very important

- FNB should never be considered a replacement for or the cause of delay in open biopsy when it is indicated.

\section{Conclusion}

The basis of treatment for any disease process depends on two parts, one is generalized and the other is disease specific. It becomes very important to attain a definitive diagnosis as the disease specific treatment yields the best results. Biopsy remains the gold standard for a definitive diagnosis. The histopathological diagnosis and the clinical diagnosis form the heart and soul of the final diagnosis. The different types of biopsies are indicated for different disease process depending on its site, location, extent, accessibility, nature and the armamentarium available. Each method has its own advantages and disadvantages. Therefore, the clinician must choose the type of biopsy method very wisely so as to reach the best diagnosis in shortest period of time.

\section{Bibliography}

1. Mota-Ramirez A., et al. "Oral biopsy in dental practice". Medicina Oral Patologia Oral y Cirugia Bucal 12 (2007): E504-E510.

2. Poh CF., et al. "Biopsy and histopathologic diagnosis of oral premalignant and malignant lesions". Journal of the Canadian Dental Association 74 (2008): 283-288.

3. Ephros H and James WD. "Oral Tissue Biopsy" (2014).

4. Rosebush MS., et al. "The Oral Biopsy: Indications, Techniques and Special Considerations". Journal of the Tennessee Dental Association 90 (2010):17-20.

5. Goyal D., et al. "An Update on precancerous lesions of oral cavity". International Journal of Medical and Dental Sciences 2.1 (2013): 70-75.

6. Gray M., et al. "The clinical effectiveness of toluidine blue dye as an adjunct to oral cancer screening in general dental practice (2000). 
7. Parlatescu I., et al. "Oral Leukoplakia - An Update". MAEDICAa Journal of Clinical Medicine 9.1 (2014): 88-93.

8. Epstein JB., et al. "Advances in the Diagnosis of Oral Premalignant and Malignant Lesions". Journal of the Canadian Dental Association 68.10 (2002): 617-621.

9. Diz P., et al. "Oral leukoplakia and erythroplakia: a protocol for diagnosis and management". EAOM-Diagnostic and therapeutic protocols Oral leukoplakia and erythroplakia (2011).

10. Mutalik S., et al. "Oral Leukoplakia- Is Biopsy at the Initial Appointment a Must?" Journal of Clinical and Diagnostic Research 8.8 (2014): ZC04-ZC07.

11. Melrose RJ., et al. "American Academy of Oral and Maxillifacial Pathology. The use of biopsy in dental practice. The position of the American Academy of Oral and Maxillofacial Pathology". General Dentistry 55.5 (2007): 457-461.

12. Mashberg A and Samit A. "Early diagnosis of asymptomatic oral and oropharyngeal squamous cancers". CA: A Cancer Journal for Clinicians 45.6 (1995): 328-351.

13. Driemel 0., et al. "Diagnosis of oral squamous cell carcinoma and its precursor lesions". JDDG: Journal der Deutschen Dermatologischen Gesellschaft 5.12 (2007): 1095-1100.

14. Collaborators from Royal College of Dental Surgeons of Ontario. "Evidence-based clinical recommendations regarding screening for oral squamous cell carcinomas". Supplement to Aug/Sept 2010 isssue of Dispatch magazine (2010).

15. Gandolfo S., et al. "Biopsy technics in oral oncology: excisional or incisional biopsy? A critical review of the literature and the author personal contribution". Minerva Stomatologica 42 (1993): 69-75.

16. Moore EG. "The Importance of biopsy Procedure”. The Journal of the American Medical Association 205 (1968): 917-920.

17. Hershita Singh., et al. "Biopsy- A vision of life". International Journal of Contemporary Medical Research 3.6 (2016): 17341737.

18. Oliver RJ., et al. "Oral biopsies: methods and applications". British Dental Journal 196 (2007): 329-333.
19. Poh CF., et al. "Fluorescence visualization detection of field alterations in tumor margins of oral cancer patients". Clinical Cancer Research 12.22 (2006): 6716-6722.

20. Avon LS and Hagen BE. "Oral Soft Tissue Biopsy: An Overview". Journal of the Canadian Dental Association 78 (2012): c75.

21. Shklar G. "The effect of manipulation and incision on experimental carcinoma of hamster buccal pouch". Cancer Research 28 (1968): 2180-2182.

22. Poh CF., et al. "Biopsy and histopathologic diagnosis of oral premalignant and malignant lesions". Journal of the Canadian Dental Association 74.3 (2008): 283-288.

23. Kinsukawa J., et al. "Dissemination of cancer cells into circulation occurs by incisional biopsy of oral squamous cell carcinoma". Journal of Oral Pathology and Medicine 29 (2000): 303-307.

24. Eisen D. "The oral mucosal punch biopsy. A report of 140 cases". Archives of Dermatological 128 (1992): 815-817.

25. Sylvie-Louise Avon DM and Klieb HB. “Oral soft-tissue biopsy: an overview". Journal of the Canadian Dental Association 78 (2012): c75.

26. Hall DL. "Oral Brush Biopsy Technique Instruction outcome for senior Dental Students". The Journal of Dental Education 4.70 (2006): 820-824.

27. Epstein JB., et al. "Analysis of oral lesion biopsies identified and evaluated by visual examination, chemiluminescence and toluidine blue". Oral Oncology 44 (2008): 538-544.

\section{Assets from publication with us}

- Prompt Acknowledgement after receiving the article

- Thorough Double blinded peer review

- Rapid Publication

- Issue of Publication Certificate

- High visibility of your Published work

Website: www.actascientific.com/

Submit Article: www.actascientific.com/submission.php

Email us: editor@actascientific.com

Contact us: +919182824667 